\title{
Implementation of Secondary Index on Cloud Computing NoSQL Database in Big Data Environment
}

\author{
Bao Rong Chang, ${ }^{1}$ Hsiu-Fen Tsai, ${ }^{2}$ Chia-Yen Chen, ${ }^{1}$ \\ Chien-Feng Huang, ${ }^{1}$ and Hung-Ta Hsu ${ }^{1}$ \\ ${ }^{1}$ Department of Computer Science and Information Engineering, National University of Kaohsiung, Kaohsiung 81148, Taiwan \\ ${ }^{2}$ Department of Marketing Management, Shu-Te University, Kaohsiung 82445, Taiwan
}

Correspondence should be addressed to Chien-Feng Huang; cfhuang15@nuk.edu.tw

Received 4 September 2014; Accepted 12 December 2014

Academic Editor: Gianluigi Greco

Copyright (C) 2015 Bao Rong Chang et al. This is an open access article distributed under the Creative Commons Attribution License, which permits unrestricted use, distribution, and reproduction in any medium, provided the original work is properly cited.

\begin{abstract}
This paper introduces the combination of NoSQL database HBase and enterprise search platform Solr so as to tackle the problem of the secondary index function with fast query. In order to verify the effectiveness and efficiency of the proposed approach, the assessment using Cost-Performance ratio has been done for several competitive benchmark databases and the proposed one. As a result, our proposed approach outperforms the other databases and fulfills secondary index function with fast query in NoSQL database. Moreover, according to the cross-sectional analysis, the proposed combination of HBase and Solr database is capable of performing an excellent query/response in a big data environment.
\end{abstract}

\section{Introduction}

Regarding big data storage $[1,2]$, the way of fast and easy data query is a concerned issue in NoSQL database. In general, NoSQL scheme $[3,4]$ is capable of supporting various data format to process the storage; yet it sacrifices the index searching function. HBase is of a NoSQL database as part of Hadoop ecosystem. It is known as the scheme of key value and usually stores the results coming out of MapReduce execution. HBase features high scalability and high flexibility, delivering a high IO performance of big data. Solr is of a blazing fast open source enterprise search engine that can quickly create index and proceed with powerful full-text search. In this paper, we are able to combine HBase and Solr to enhance the secondary index function for HBase. After the success of this combination, we go for a series of stress tests using several testing items and then make the performance comparison between the proposed one and the other benchmark databases. Finally, a cost effectiveness evaluation called Cost-Performance ratio (C-P ratio) [5] has been done for a variety of databases. As a result, the assessment about $\mathrm{C}-\mathrm{P}$ ratio will be analyzed and discussed for all of databases mentioned in this paper. Based on the cross-sectional data analysis [6], it will explore the performance of data access in NoSQL database in a big data environment as well.

For key-value database, it allows the application to store its data in a schema-less way. The data could be stored in a data type of a programming language or an object. There is no need for a fixed data model. Key-value storing divides many categories, like eventually consistent (always keeps the newest result if there is no update), hierarchical (can use the parent's attributes), cache in RAM (key value stored in memory, hash stored in cache, and hash used to present keyvalue index; time complexity is $\mathrm{O}(1)$ ), solid state or rotating disk (like Google Bigtable which is used in solid state disk to enhance IO access speed), and ordered (with key-value pairs which can sort keys or values). For tabular database, it is a database that is structured in a tabular form. It arranges data elements in vertical columns and horizontal rows. Each cell is formed by the intersection of a column and row. Each row and column are uniquely numbered to make it orderly and efficient. This type of database has a virtually infinite range for mass data storage. Structuring data in tabular form may be the oldest method used. It is also simple. Tabular 
TABLE 1: NoSQL database benchmark on 5 criteria.

\begin{tabular}{|c|c|c|c|c|c|}
\hline Database & Performance & Scalability & Flexibility & Complexity & Functionality \\
\hline Key-value store & High & High & High & Low & Variable \\
\hline Column store & High & High & Moderate & Low & Minimum \\
\hline Document store & High & Variable & High & Low & Variable \\
\hline Graph database & Variable & Variable & High & High & Graph theory \\
\hline Relational database & Variable & Variable & Low & Moderate & Relational algebra \\
\hline
\end{tabular}

database has several properties. They share the same set of properties per record. This means that every row has the same set of column titles. They access records through identifiers. Each table in a tabular database contains a particular set of related information that is connected with the database subject through key fields, which describe each record (row) so that, in the event of a query, the system can quickly locate the record. There are several famous databases of this type, like Google Bigtable, Apache Accumulo, Apache HBase, and Hypertable. For column store database, it stores data tables as sections of columns of data rather than rows of data. For RDBMS, rows are commonly used; the column store database has the advantage of aggregating computed data over large numbers of similar data items. Column store is used in data warehouse and CRM system. Using column store database, the system can evaluate which columns are being accessed and retrieved only if values are requested from the specific columns. For NoSQL database, each mechanism has different uses, and a famous database can have many properties, like Google Bigtable. It owns solid state disk key-value type and tabular type. For this study, HBase is a column-store database. It has an easy method to use, and its performance as well as the scalability is better than the others. Table 1 explains the performance of each type of database over 5 criteria.

The following paragraphs of this paper are arranged as follows. In Section 2, combination of NoSQL database and enterprise search platform will be described. The way to system assessment is given in Section 3. The experimental results and discussion will be obtained in Section 4. Finally, we drew a brief conclusion in Section 5 .

\section{Combination of NoSQL Database and Enterprise Search Platform}

This paper studies how the combination of HBase and Solr runs in big data environment based on cloud computing platform. All of application programs were installed in a Linux-based operating system. HBase is placed over Hadoop HDFS system. Thus, HBase can be attached to Hadoop after the core parts of Hadoop have been installed in a physical machine such as MapReduce and HDFS. Solr can operate independently without any support from any other applications. With the corporation with Solr, HBase can easily create index. On the other hand, Solr is able to provide GUI interface for user's operation. The procedure to establish the combination of two applications can be listed as follows.
(1) Install Linux $\mathrm{O} / \mathrm{S}$ on every host, connect them together via SSH, and deploy JVM to every host to achieve a Linux cluster environment.

(2) Establish master and slave nodes and start them up. Master node shall deploy Hadoop to slave nodes. This has Hadoop done in every host in a cluster environment [7-9].

(3) After deploying Hadoop and ZooKeeper to cluster, we need to confirm the start-up of Hadoop and ZooKeeper services. We are able to give jps instruction at terminal to check whether or not the services are running normally. After that, we establish HBase service [10-13] within Hadoop.

(4) When procedure \#3 has been done, web browser is used to view the start-up of Hadoop and HBase services. Key in http://localhost:50030/, 50040, 50070, and 60010 is used to check each node if operating normally.

(5) Before we get Solr started, we need to modify the execution parameters in solrconfig.xml, which is a configuration file within ./solr-version/examples/ solr/collection1/conf/. We have to determine the Solr whether or not setting input word string to act as an index, content storage, and data format. Apache Solr needed http web container to get it started, for example, either Apache Tomcat or Apache Jetty. Here, we chose Jetty because of the default setting. After setting up, we key in "java -jar start, jar" to start up Solr in terminal. Finally, we got Solr's address, which is http://localhost:8983/.

(6) Since HBase cannot support automatically generated row key, several big data files shall be modified in advance. We need to design a unique and complex rowkey which corresponds to a large number of rows (up to ten million rows). In this study, we chose the American Yellow Page as data source. Our data combination is "rowkey-category-shop name-telephoneprovince-address" with a total of 6 columns. These data files have to translate into CSV format, and ", symbols are used to separate each column.

(7) The CSV file is uploaded to Hadoop file system, and these files are imported to HBase as full-text input via the special tool, "bulk load tool" [14]. We need to check the data integrity in HBase after data importing. 


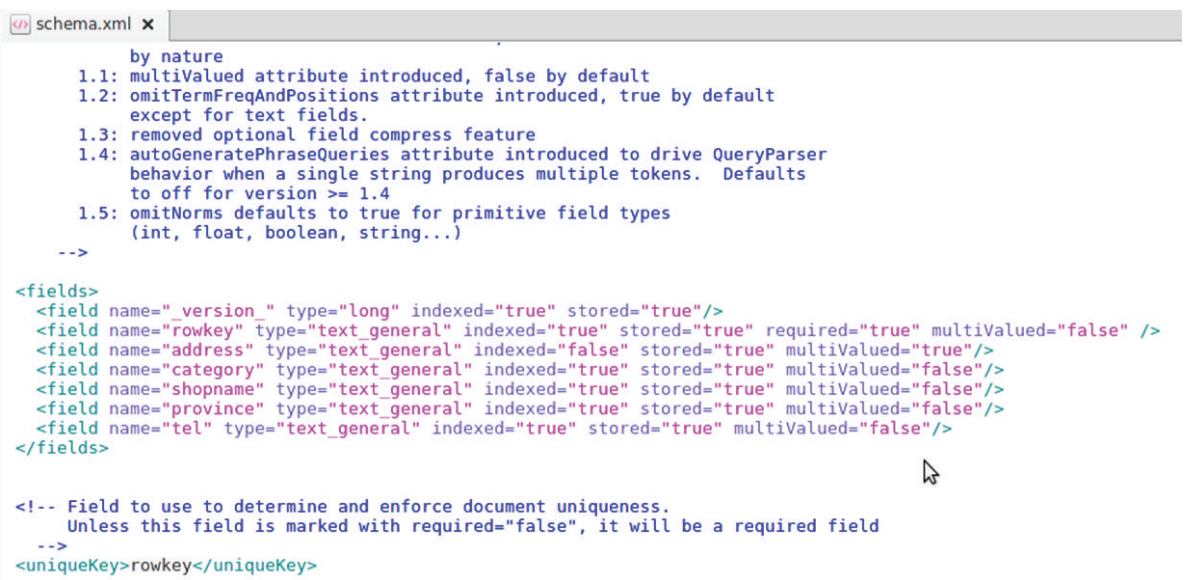

FIGURE 1: Apache Solr configuring file for indexing.

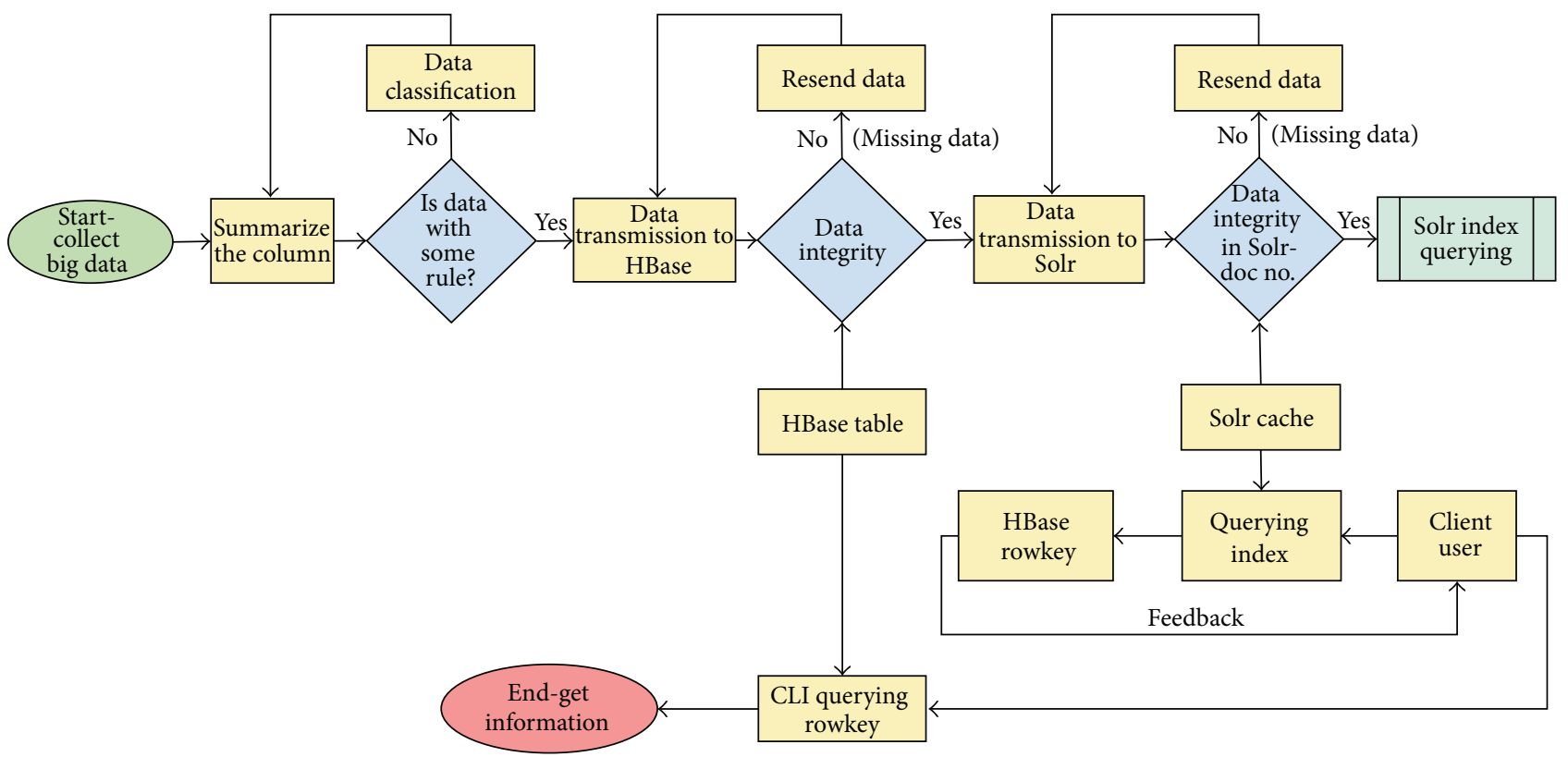

FIGURE 2: Flowchart of HBase together with Solr to implement secondary index operation.

(8) Then, we use HBase output API and Apache HTTP API to transfer the document to Solr from HBase [1517]. After the transmission, the indexes are created and the content is saved in memory in Solr, that is, the schema as defined and shown in Figure 1. We can use web browser to check the amount of documents in Solr. Data in a row represent a document. We can use query function to search our keyword (Secondary index or more) and reversely to search the primary index in Solr. We may be able to apply filter function to improve the precision of search results.

(9) After finishing the setup of the proposed system, we chose some other benchmarks to compare with the proposed one in the experiment. After the experiment, we are able to give a kind of assessment on those, for instance a cost effectiveness evaluation.
In Figure 2, a flowchart represents HBase together with Solr to implement secondary index operation.

\section{System Assessment}

In terms of the performance evaluation, we have initially tested the time for data read/write to a variety of databases, such as Apache HBase, Cassandra, Huawei HBase, Solandra, and Lily Project. Next, the time for data transfer to Solr from the databases mentioned above has to be recorded. Finally, the response time for the query function performed in Solr needed to be measured as well. According to four tests on data write, data read, document transfer, and query/response to any of databases as mentioned above, first of all we have to measure a single datum access time taking a number of different data size as shown in (1), where $t_{s_{i j k}}$ represents a single datum access time, for a single run $t_{i j k}$ stands for 


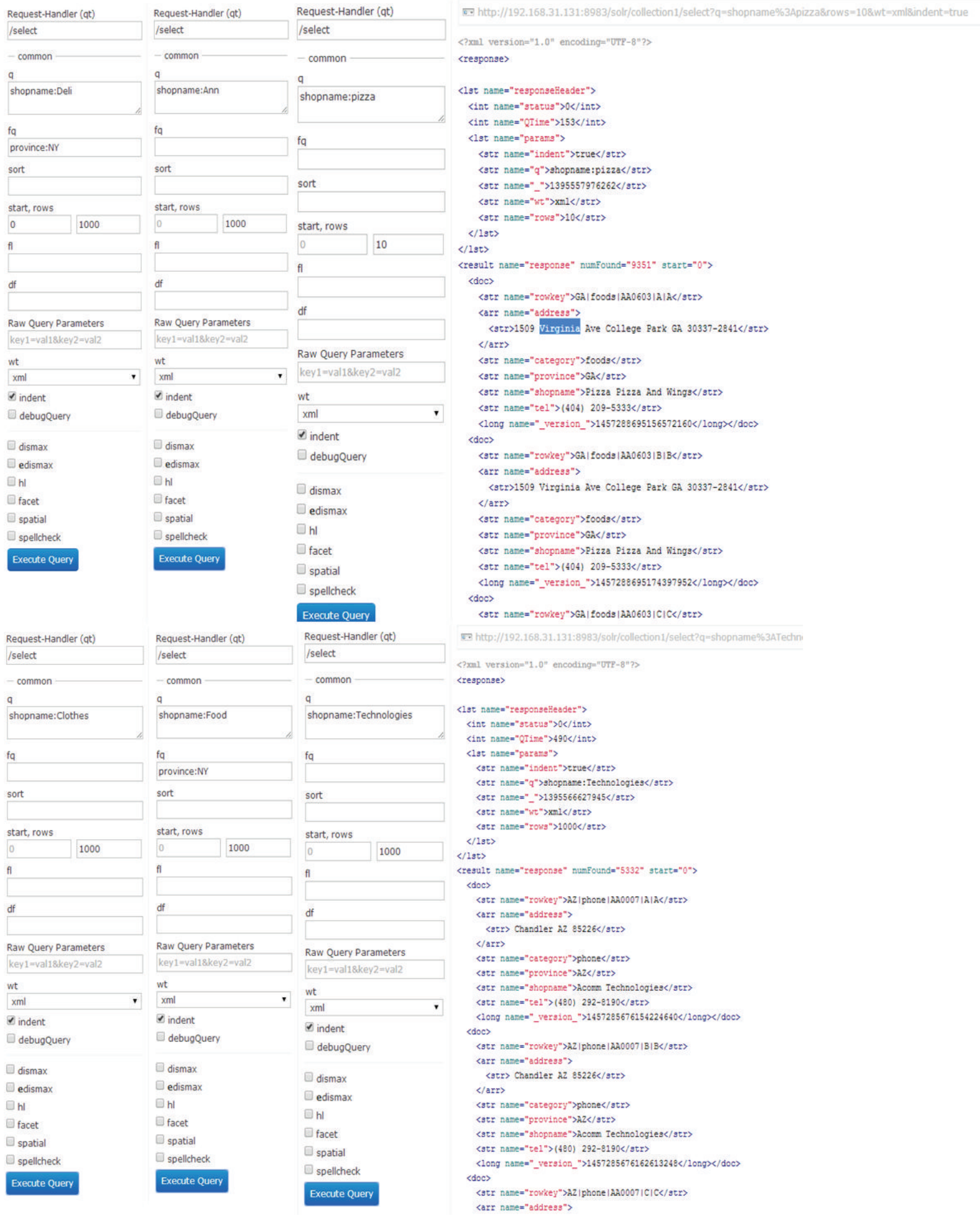

Figure 3: Latency under stress test for Solr (presenting 6 windows). 
measured total time for a specific data size at a certain database, and $N_{i k}$ means a specific data size. In (2), $\bar{t}_{s_{i j k}}$ represents average time of a single datum access and $w_{i}$ stands for the respective weight factor for $t_{s_{i j k}}$. A normalized performance index for a specific database at a certain test can be obtained as shown in (3), where $\overline{\mathrm{PI}}_{j k}$ represents a normalized performance index. After that, we have evaluated the weighted average of normalized performance index and it turned out to be the performance index [18] for each database as shown in (4), where $\mathrm{PI}_{j}$ represents performance index, $\mathrm{SF}_{1}$ stands for scale factor $\# 1, W_{k}$ is the respective weight, and $\overline{\mathrm{PI}}_{j k}$ means a normalized performance index. In order to assess the cost effectiveness evaluation, we need to calculate total cost of ownership [19] in (5), showing the expenditure of money in the implementation of secondary index function for NoSQL database, where $\mathrm{HC}_{a}$ presents hardware cost, $S_{b}$ stands for software cost, $\mathrm{RCAW}_{c}$ means repairing cost after the warranty, $\mathrm{DTC}_{d}$ is downtime cost, and $\mathrm{EUC}_{e}$ explains extra upgrade cost. The monetary value of total cost of ownership may vary with location, market, and tax. Thus, a higher cost, for example, might be obtained in US and a lower cost in Taiwan. In the system assessment, a typical cost effectiveness evaluation called C-P ratio has been introduced here to do the assessment in (6), where $\mathrm{CP}_{j g}$ is C-P ratio, $\mathrm{SF}_{2}$ stands for scale factor \#2, and $\mathrm{TCO}_{j g}$ means total cost of ownership as well as subscript $j$ that represents various data center and $g$ that stands for a certain period of time. Consider the following:

$$
t_{s_{i j k}}=\frac{t_{i j k}}{N_{i k}},
$$

where $i=1,2, \ldots, l, j=1,2, \ldots, m$, and $k=1,2, \ldots, n$,

$$
\bar{t}_{s_{j k}}=\sum_{i=1}^{l} w_{i} \cdot t_{s_{i j k}}
$$

where $j=1,2, \ldots, m, k=1,2, \ldots, n$, and $\sum_{i=1}^{l} w_{i}=1$,

$$
\overline{\mathrm{PI}}_{j k}=\frac{1 / \bar{t}_{s_{j k}}}{\operatorname{Max}_{h=1,2, \ldots, m}\left(1 / \bar{t}_{s_{h k}}\right)},
$$

where $j=1,2, \ldots, m$ and $k=1,2, \ldots, n$,

$$
\mathrm{PI}_{j}=\mathrm{SF}_{1} \cdot\left(\sum_{k=1}^{n} W_{k} \cdot \overline{\mathrm{PI}}_{j k}\right),
$$

where $j=1,2, \ldots, m, k=1,2, \ldots, n, \mathrm{SF}_{1}=10^{2}$, and $\sum_{k=1}^{n} W_{k}=1$,

$$
\mathrm{TCO}_{j g}=\sum_{a} \mathrm{HC}_{a}+\sum_{b} S_{b}+\sum_{c} \mathrm{RCAW}_{c}+\sum_{d} \mathrm{DTC}_{d}+\sum_{e} \mathrm{EUC}_{e},
$$

where $j=1,2, \ldots, m$ and $g=1,2, \ldots, o$,

$$
\mathrm{CP}_{j g}=\mathrm{SF}_{2} \cdot \frac{\mathrm{PI}_{j}}{\mathrm{TCO}_{j g}},
$$

where $j=1,2, \ldots, m, g=1,2, \ldots, o$, and $\mathrm{SF}_{2}=10^{4}$.

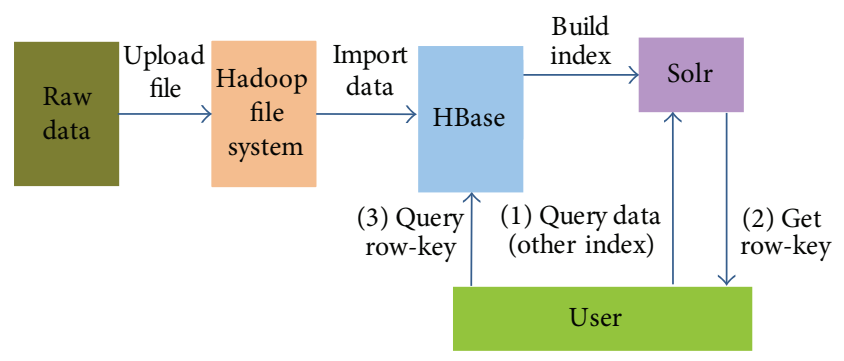

FIGURE 4: Implementation procedure.

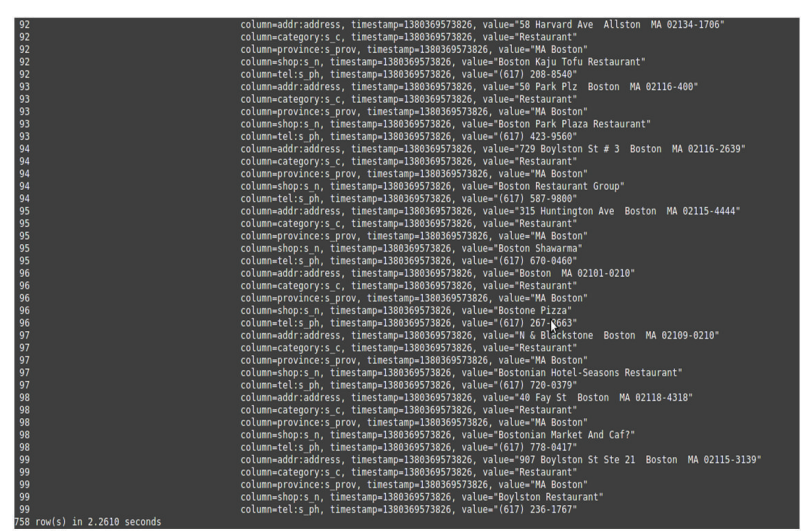

Figure 5: Scanning a table in HBase using CLI.

In order to examine the stability and reliability of NoSQL database secondary index function, a stress test of data retrieval in Solr has been taken in a big data environment. Technically speaking, this test generated up to 20 threads (20 windows) to respond to 10 to 1000 queries and we had checked the latency (time interval) simultaneously. The key index in every query was different as shown in Figure 3. Clearly, the result would indicate the response time for the query in Solr and explain what correlation between the amount of windows and the latency was found.

\section{Experimental Results and Discussion}

There are a few experiments and a discussion presented in the following subsections.

4.1. Data Transfer and Data Integrity Checking. In regard to implementation procedure as shown in Figure 4, which indicated data transfer from HDFS to HBase and/or from HBase to Solr, there are risks of losing data during the transition. We have to verify the data integrity in HBase inner table and the amount of input documents in Solr. For examining HBase, we checked inner table using the command "scan table-name" in CLI as shown in Figure 5. In Figure 6, the document transfer from HBase to Solr has been done using the command in CLI. For examining Solr, we checked our input document amount in Solr using web interface as shown in Figure 6. Furthermore, in terms of the performance evaluation, the time for data writing/reading in every database has been measured as listed in Tables 2 and 3 . 
TABLE 2: Time for writing data to database (unit: sec.).

\begin{tabular}{lccccc}
\hline Data size & HBase + Solr & Cassandra & Huawei HBase & Solandra & Lily Project \\
\hline $10^{4}$ & 23 & 110.4 & 23 & 120 & 23 \\
$10^{5}$ & 23.2 & 1109.2 & 23 & 1215 & 24 \\
$10^{6}$ & 123.4 & 11211.3 & 125 & 11253 \\
$10^{7}$ & 388.5 & 113157.7 & 390 & 113189 & 137 \\
\hline
\end{tabular}

TABLE 3: Time for reading data from database (unit: sec.).

\begin{tabular}{lccccc}
\hline Data size & HBase + Solr & Cassandra & Huawei HBase & Solandra & Lily Project \\
\hline $10^{4}$ & 27.2 & 27.6 & 30 & 29 & 27 \\
$10^{5}$ & 266.5 & 270.7 & 269 & 288.5 & 273 \\
$10^{6}$ & 2572.2 & 2614.2 & 2589.7 & 2735 & 2566 \\
$10^{7}$ & 24312 & 24701 & 24479 & 24988 & 24385 \\
\hline
\end{tabular}

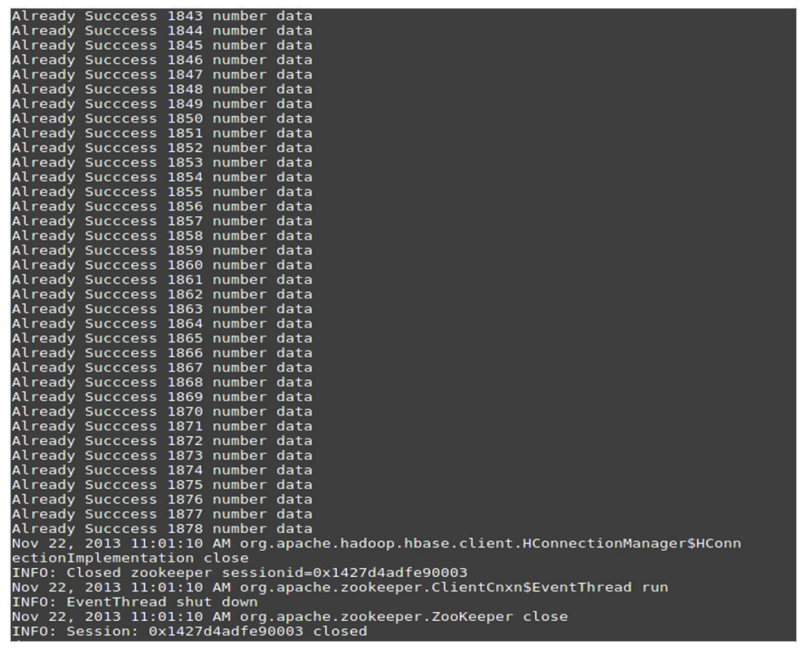

Figure 6: Importing data to Solr from HBase.

Time for data transfer to Solr from every database has been recorded as listed in Table 4.

Speaking of data import to HBase, we adopted a bulk-load tool with MapReduce computing to transfer the original file into HBase because this tool is capable of handling a large amount of data in the way of fast and smoothly transferring. For Solr, a program with specific port at Solr and designated HBase API has activated to quickly transfer documents to Solr from HBase where a java client to access Solr called Solrj has logged into the http server, that is Solr, to respond swiftly to the connection and deliver the on-line document to http server. This also demonstrates an efficient way to realize a fast document transfer based on a client-server model for a huge amount of data. Alternatively, the other choice is that HBase coprocessor may launch a process to do the batch update frequently. However, HBase coprocessor is not stable because it is still in the developing phase.

4.2. Querying Function and Performance Index. Once the document transfer from HBase to Solr has been done completely, the data are available in Solr and we could check the

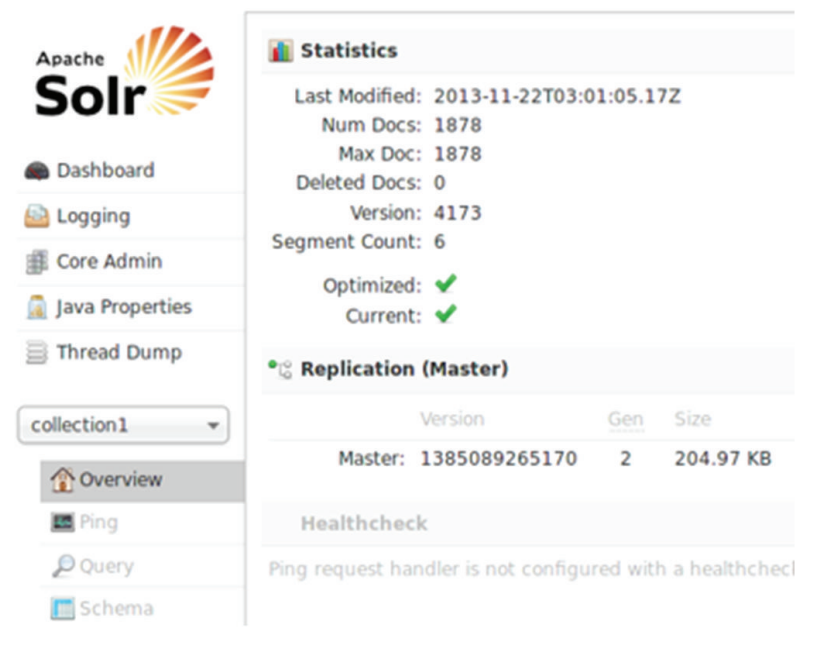

FIgURE 7: Presentation of Imported data in Solr using GUI.

amount of document in Solr as shown in Figure 7. In order to verify the secondary index function in the combination of HBase and Solr, we launched the query test in Solr as shown in Figure 8, where we can check the information about the related operations on the web. Solr provides normal search, filtering search, spatial search, and other more search functions. For example, we did a search using the shop-name field that included "Food" as its keyword, and 1000 results appeared filtering the province tag with "NY." We keyed in "shopname:Food" in "q" field, inputted "province:NY" in "fq" field, and gave 1000 in rows field. Figure 8 has shown the operation of query. In Table 5, the response time for the query function performed in Solr has also been marked. Besides, average time-consuming on data read/write, document transfer, and query function is eventually obtained as listed in Table 6. After that, according to (4), we are able to evaluate the performance index for each database over a 5-year period of time as shown in Table 7.

4.3. Assessment. In the system assessment, we first analyze total cost of ownership (TCO) according to several items such as hardware cost, staff cost, software cost, repair cost 


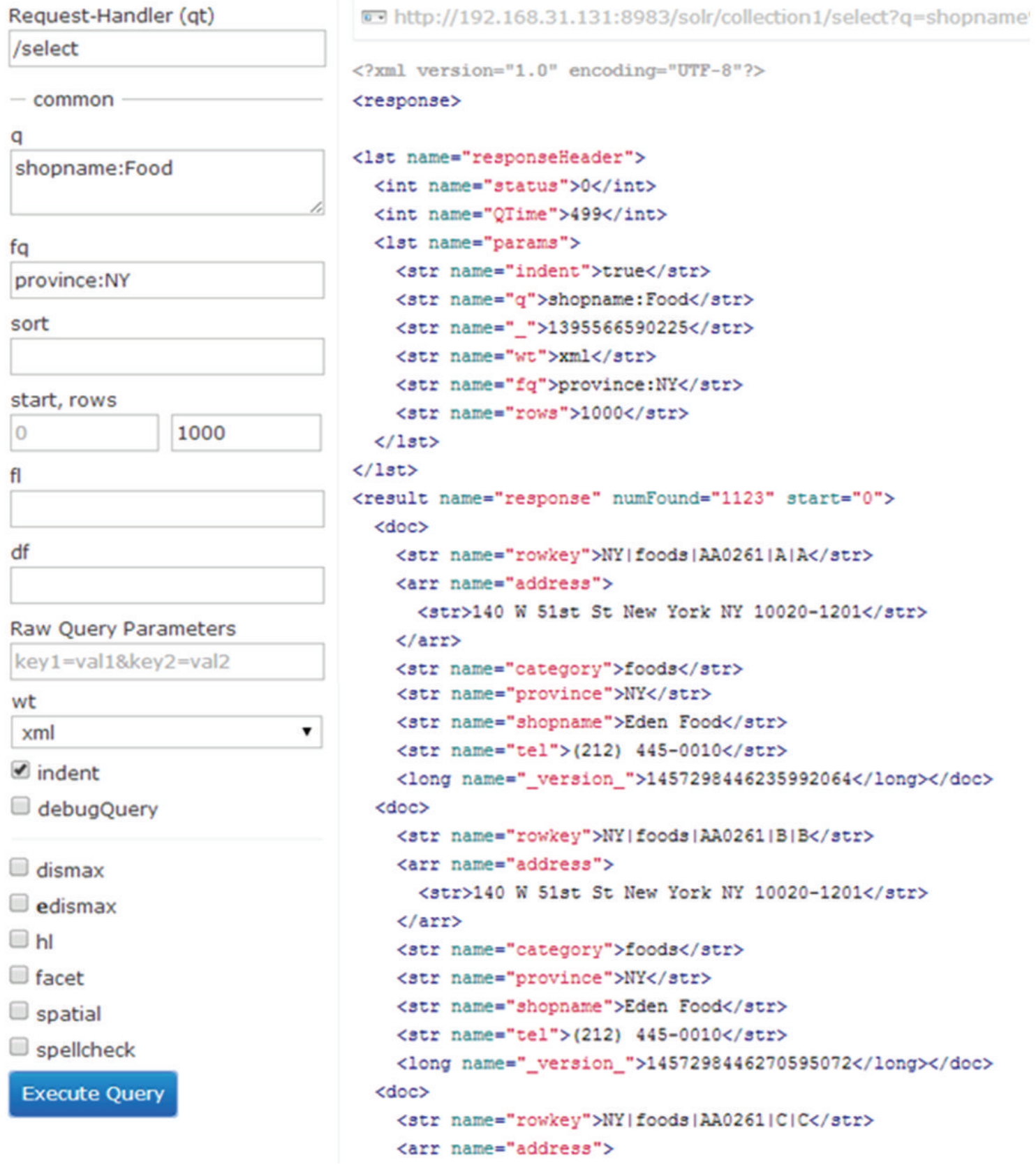

FIgURE 8: Response to a query in Solr using GUI.

after warranty, downtime cost, and extra upgrade cost. A summary of TCO has been shown in Table 7. Here we estimated that hardware cost for two computers is $\$ 2666$. Then, we assumed that the maintenance bill is $\$ 13000$ every year for Hadoop together with HBase, Solr maintenance cost is approximately $\$ 300$ per year, and for Cassandra it would be $\$ 10300$ every year. Accordingly, we do the same maintenance estimation as the above-mentioned applications for Solandra and Lily Project because they are just the combination of the above applications. All of software cost is totally free due to open source. For hardware maintenance after warranty, we assumed that all the devices had the same risk of breakdown, and thus the chance of device breakdown in the 4 th year was about $25 \%$, while in the 5 th year it will be $50 \%$ chance. For the software upgrade cost, there is no charge because of open source. Regarding downtime cost, we assumed that one application will cost $\$ 20$ per year and the total cost would depend on the amount of software. Table 8 gives a summary of the total cost of ownership for this study. As for the system assessment, C-P ratio evaluation according to (6) for all of databases will yield a summary of those over a 5 -year period of time as listed in Table 9.

4.4. Stress Test and Discussion. The issue about the stability and reliability of NoSQL database secondary index function has been concerned and hence a stress test of data retrieval 
TABLE 4: Document transfer time from database to Solr (unit: sec.).

\begin{tabular}{lccccc}
\hline Data size & HBase + Solr & Cassandra & Huawei HBase & Solandra & Lily Project \\
\hline $10^{4}$ & 109 & 120 & 115 & 123 & 115 \\
$10^{5}$ & 1121 & 1130 & 1125 & 1154 & 1130 \\
$10^{6}$ & 11105 & 11286 & 11173 & 11330 & 11186 \\
$10^{7}$ & 108055 & 112806 & 112347 & 113105 & 112395 \\
\hline
\end{tabular}

TABLE 5: Response time for querying function performed in Solr (unit: sec.).

\begin{tabular}{lccccc}
\hline Data size & HBase + Solr & Cassandra & Huawei HBase & Solandra & Lily Project \\
\hline $10^{4}$ & 0.15 & 0.91 & 45 & 2 & 7 \\
$10^{5}$ & 0.5 & 11.12 & 288 & 5 & 1 \\
$10^{6}$ & 2 & 143.1 & 547 & 15 & 10 \\
$10^{7}$ & 10 & 2011.13 & 1867 & 60 & 45 \\
\hline
\end{tabular}

TABLE 6: Average time of a single datum access (unit: sec.).

\begin{tabular}{lccccc}
\hline Operation & HBase + Solr & Cassandra & Huawei HBase & Solandra & Lily Project \\
\hline Data write & 0.000673563 & 0.011164768 & 0.0006735 & 0.011680475 & 0.00067955 \\
Data read & 0.0025971 & 0.002637825 & 0.0026819 & 0.0027547 & 0.002608625 \\
Document transfer & 0.011005125 & 0.01146665 & 0.011289425 & 0.011620125 & 0.011306375 \\
Query/response & 0.00000575 & 0.000136603 & 0.002028425 & 0.00007275 & 0.000041125 \\
\hline
\end{tabular}

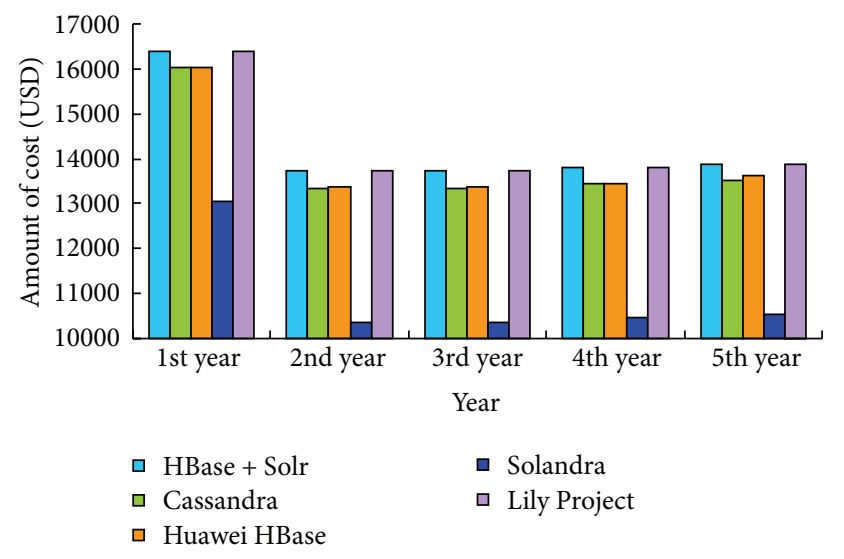

Figure 9: Total cost of ownership among various databases over a 5 -year period.

in Solr has been taken in a big data environment. In this test, there are up to 20 threads (20 windows) used to accept the number of queries from 10 to 1000 and in the meantime the latency (time interval) has been counted. The key index in every query was different as shown in Figure 3. Table 10 has listed the summary of latency and we have examined the results afterward. In the test from the statistics point of view, the amount of opening windows obviously did not affect the length of latency occurring in the query in Solr. The stability and reliability of NoSQL database secondary index function have been verified because all of queries had responded in 5 seconds during the stress test.

It noted that performance indexes for five databases have been listed in Table 7 and they are time-invariant. In Figure 9,
TABle 7: Performance index.

\begin{tabular}{lc}
\hline Database & Performance index \\
\hline HBase + Solr & 99 \\
Cassandra & 51 \\
Huawei HBase & 73 \\
Solandra & 50 \\
Lily Project & 77 \\
\hline
\end{tabular}

TABLE 8: Total cost of ownership over a 5-year period (unit: USD).

\begin{tabular}{lccccc}
\hline Database & 1st year & 2nd year & 3rd year & 4th year & 5th year \\
\hline HBase + Solr & 16393.3 & 13726.7 & 13726.7 & 13804.1 & 13877.9 \\
Cassandra & 16020 & 13353.3 & 13353.3 & 13430.8 & 13504.6 \\
Huawei & 16040 & 13373.3 & 13373.3 & 13450.8 & 13629.9 \\
Solandra & 13040 & 10373.3 & 10373.3 & 10450.8 & 10524.6 \\
Lily Project & 16393.3 & 13726.7 & 13726.7 & 13804.1 & 13877.9 \\
\hline
\end{tabular}

TABLE 9: C-P ratio over a 5-year period.

\begin{tabular}{lccccc}
\hline Database & 1st year & 2nd year & 3rd year & 4th year & 5th year \\
\hline HBase + Solr & 61.00 & 72.85 & 72.85 & 72.44 & 72.06 \\
Cassandra & 31.94 & 38.32 & 38.32 & 38.10 & 37.89 \\
Huawei & 45.92 & 55.07 & 55.07 & 54.76 & 54.04 \\
Solandra & 38.85 & 48.84 & 48.84 & 48.48 & 48.14 \\
Lily Project & 47.27 & 56.46 & 56.46 & 56.14 & 55.84 \\
\hline
\end{tabular}

the total cost of ownership for our proposed approach has varied from year to year where it goes down dramatically and goes up slowly over a 5-year period. Accordingly, C-P ratio of the proposed approach goes up abruptly and almost 
TABLE 10: Latency under stress test (unit: sec) (Win.: window).

\begin{tabular}{lcccccccccc}
\hline Query & Win. & Win. & Win. & Win. & Win. & Win. & Win. & Win. & Win. & Win. \\
\hline 10 & $\# 1$ & $\# 2$ & $\# 3$ & $\# 4$ & $\# 5$ & $\# 6$ & $\# 7$ & $\# 8$ & $\# 9$ \\
100 & 0.15 & 0.1 & 0.2 & 0.2 & 0.1 & 0.15 & 0.16 & 0.15 & 0.2 & 0.2 \\
1000 & 1 & 1 & 0.8 & 1 & 1 & 0.8 & 1 & 1 & 1 \\
\hline & 3 & 4 & 3 & 4 & 3 & 4 & 3 & 4 & 5 & 4 \\
\hline 10 & $\# 11$ & $\# 12$ & $\# 13$ & $\# 14$ & $\# 15$ & $\# 16$ & $\# 17$ & $\# 18$ & $\# 19$ & $\# 20$ \\
100 & 0.15 & 0.15 & 0.15 & 0.15 & 0.2 & 0.2 & 0.16 & 0.15 & 0.2 & 0.2 \\
1000 & 1.2 & 0.8 & 1.1 & 1 & 1.1 & 1 & 1.2 & 1 & 1.1 & 1.2 \\
\hline & 3 & 4 & 3 & 4 & 5 & 4 & 4 & 4 & 5 \\
\hline
\end{tabular}

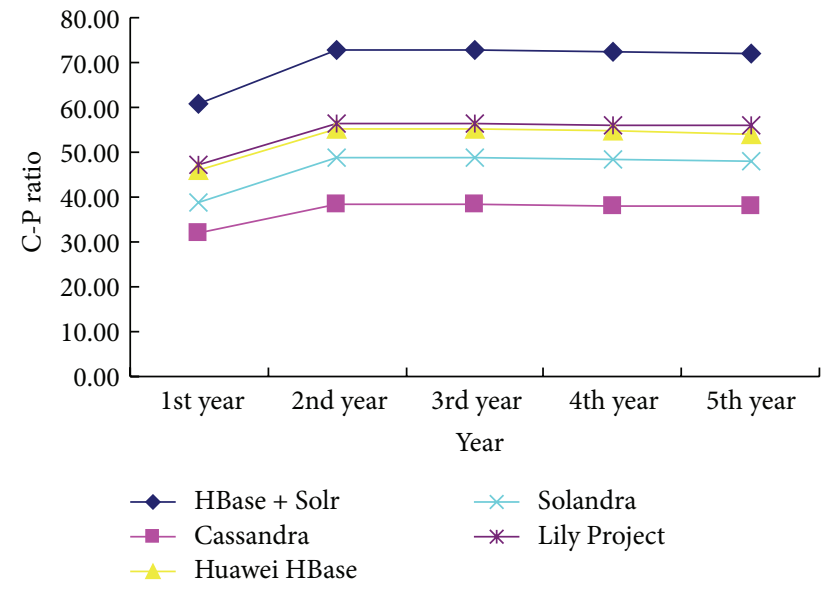

FIGURE 10: C-P ratio among various databases over a 5-year period.

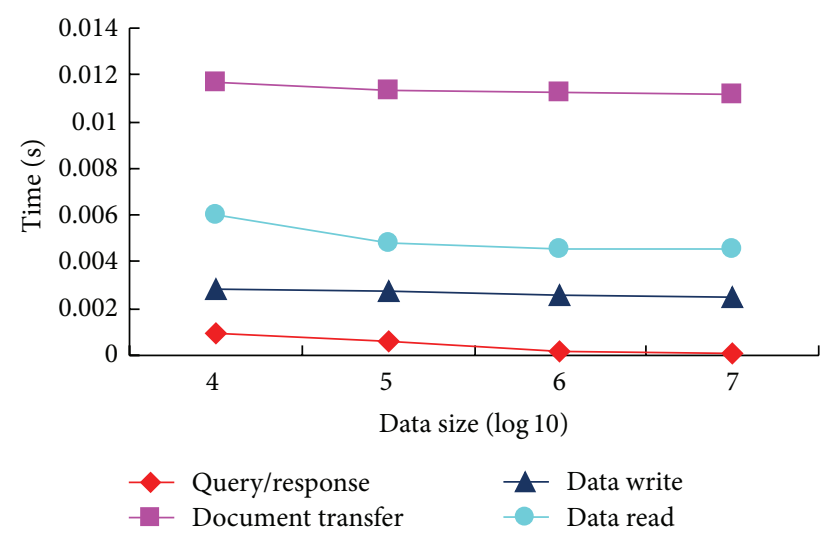

FIgURE 11: Average time of a single datum access in a certain database.

maintains the same level afterward as shown in Figure 10. Consequently, according to C-P ratio, our proposed approach outperforms the others during this period, as listed in Table 9. This has verified that our proposed approach has been realized successfully and performed significantly for a NoSQL secondary index function and fast query.

There are four tests about the function of data read, data write, document transfer, and query/response, as mentioned above in this paper to measure the average time of a single datum access in a certain database as listed in Tables 2 to 4. What we are interested in is to figure out whether the average time of a single datum access may be varied with data size or not for these functions. As shown in Figure 11, the cross-sectional data analysis [6] gave that it takes least time on the function of query/response when comparing with the other functions. The average time reduces dramatically as data size increases because the hit rate of data retrieval goes up rapidly in memory cache and concurrently the response time shrinks sharply. This figure illustrates that NoSQL database with secondary index function can achieve an excellent performance in query/response of a certain database, especially in a big data environment.

\section{Conclusion}

This paper introduces the combination of NoSQL database HBase and enterprise search platform Solr to realize the secondary index function with fast query. In the assessment, a cost effectiveness evaluation called C-P ratio has been done among several competitive benchmark databases and the proposed one. As a result, our proposed approach outperforms the other databases and fulfills secondary index function with fast query in NoSQL database. Besides, a stress test has been taken to verify the stability and reliability of the proposed approach. Finally, according to the cross-sectional analysis, the proposed combination of HBase and Solr database is capable of performing an excellent query/response in a big data environment.

\section{Conflict of Interests}

The authors declare that there is no conflict of interests regarding the publication of this paper.

\section{Acknowledgment}

This work is supported by the Ministry of Science and Technology, Taiwan, Republic of China, under Grant no. MOST 103-2221-E-390-011.

\section{References}

[1] D. Howe, M. Costanzo, P. Fey et al., "Big data: the future of biocuration," Nature, vol. 455, no. 7209, pp. 47-50, 2008. 
[2] A. Jacobs, "The pathologies of big data," Communications of the ACM-A Blind Person's Interaction with Technology, vol. 52, no. 8, pp. 36-44, 2009.

[3] R. Cattell, "Scalable SQL and NoSQL data stores," ACM SIGMOD Record, vol. 39, no. 4, pp. 12-27, 2010.

[4] J. Pokorny, "NoSQL databases: a step to database scalability in web environment," International Journal of Web Information Systems, vol. 9, no. 1, pp. 69-82, 2013.

[5] B. R. Chang, H.-F. Tsai, C.-M. Chen, and C.-F. Huang, "Analysis of virtualized cloud server together with shared storage and estimation of consolidation ratio and TCO/ROI," Engineering Computations, vol. 31, no. 8, pp. 1746-1760, 2014.

[6] C.-C. Lee and C.-P. Chang, "Energy consumption and economic growth in Asian economies: a more comprehensive analysis using panel data," Resource and Energy Economics, vol. 30, no. 1, pp. 50-65, 2008.

[7] P. Zhou, J. Lei, and W. Ye, "Large-scale data sets clustering based on MapReduce and Hadoop," Journal of Computational Information Systems, vol. 7, no. 16, pp. 5956-5963, 2011.

[8] J. K. Chiang, "Authentication, authorization and file synchronization for hybrid cloud-the development centric to google apps, hadoop and linux local hosts," Journal of Internet Technology, vol. 14, no. 7, pp. 1141-1148, 2013.

[9] J. Leverich and C. Kozyrakis, "On the energy (in) efficiency of Hadoop clusters," ACM SIGOPS Operating Systems Review, vol. 44, no. 1, pp. 61-65, 2010.

[10] T. White, Hadoop: The Definitive Guide, O'Reilly Media, Sebastopol, Calif, USA, 2009.

[11] N. Dimiduk, HBase in Action, Manning Publications, Greenwich, UK, 2012.

[12] Y. Jiang, HBase Administration Cookbook, Packt Publishing, Birmingham, UK, 2012.

[13] C. Boja, A. Pocovnicu, and L. Batagan, "Distributed parallel architecture for big data," Informatica Economica, vol. 16, no. 2, pp. 116-127, 2012.

[14] J. Dean and S. Ghemawat, "MapReduce: simplified data processing on large clusters," Communications of the ACM, vol. 51, no. 1, pp. 107-113, 2008.

[15] M. Hausenblas and J. Nadeau, "Apache drill: interactive ad-hoc analysis at scale," Big Data, vol. 1, no. 2, pp. 100-104, 2013.

[16] R. Kuc, Apache Solr 4 Cookbook, Packt Publishing, Birmingham, UK, 2013.

[17] T. Grainger and T. Potter, Solr in Action, Manning Publications, Greenwich, UK, 2014.

[18] B. R. Chang, H.-F. Tsai, and C.-M. Chen, "High-performed virtualization services for in-cloud enterprise resource planning system," Journal of Information Hiding and Multimedia Signal Processing, vol. 5, no. 4, pp. 614-624, 2014.

[19] B. R. Chang, H.-F. Tsai, and C.-M. Chen, "Evaluation of virtual machine performance and virtualized consolidation ratio in cloud computing system," Journal of Information Hiding and Multimedia Signal Processing, vol. 4, no. 3, pp. 192-200, 2013. 

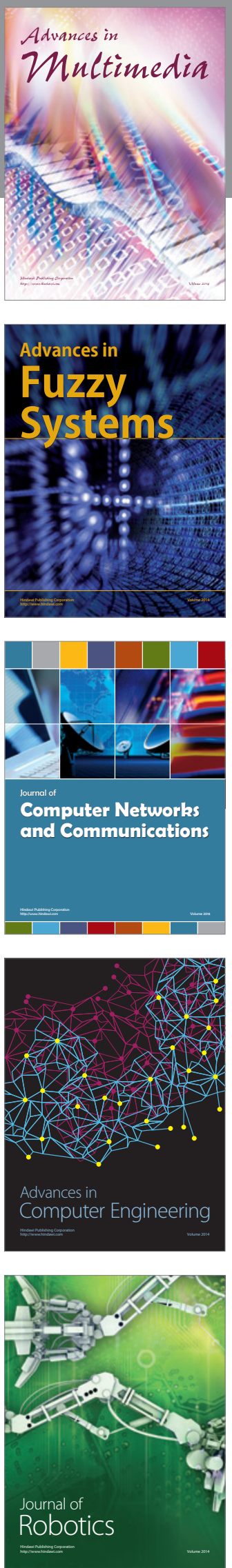

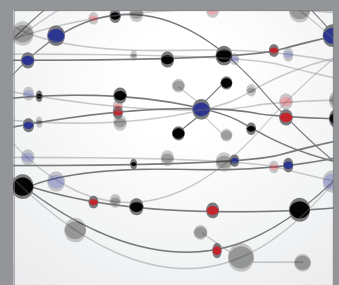

The Scientific World Journal
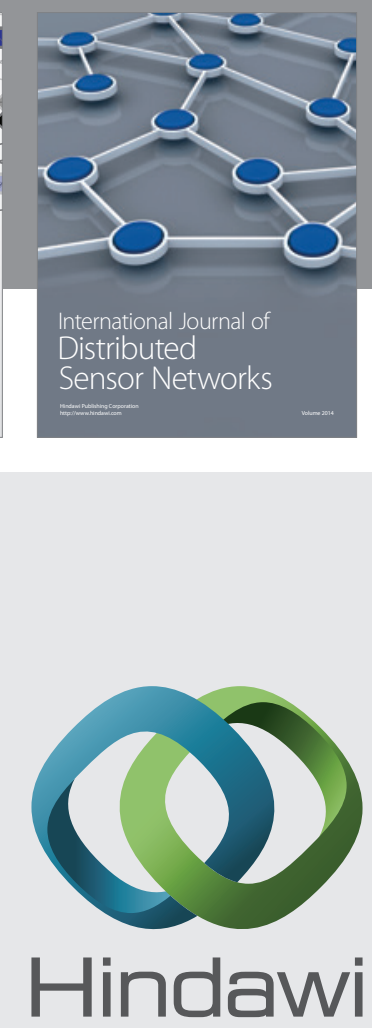

Submit your manuscripts at

http://www.hindawi.com
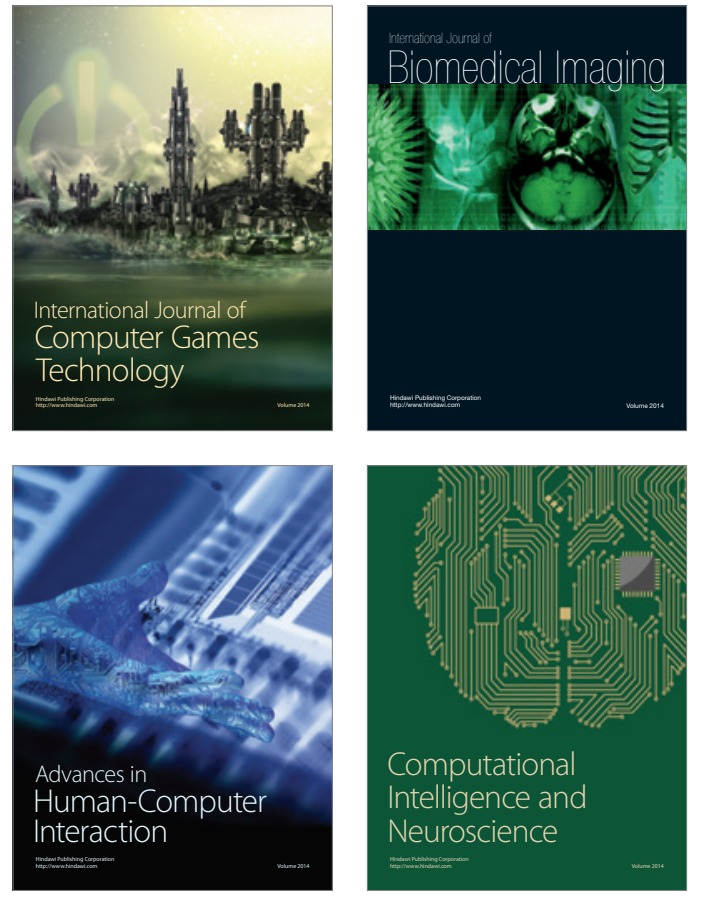
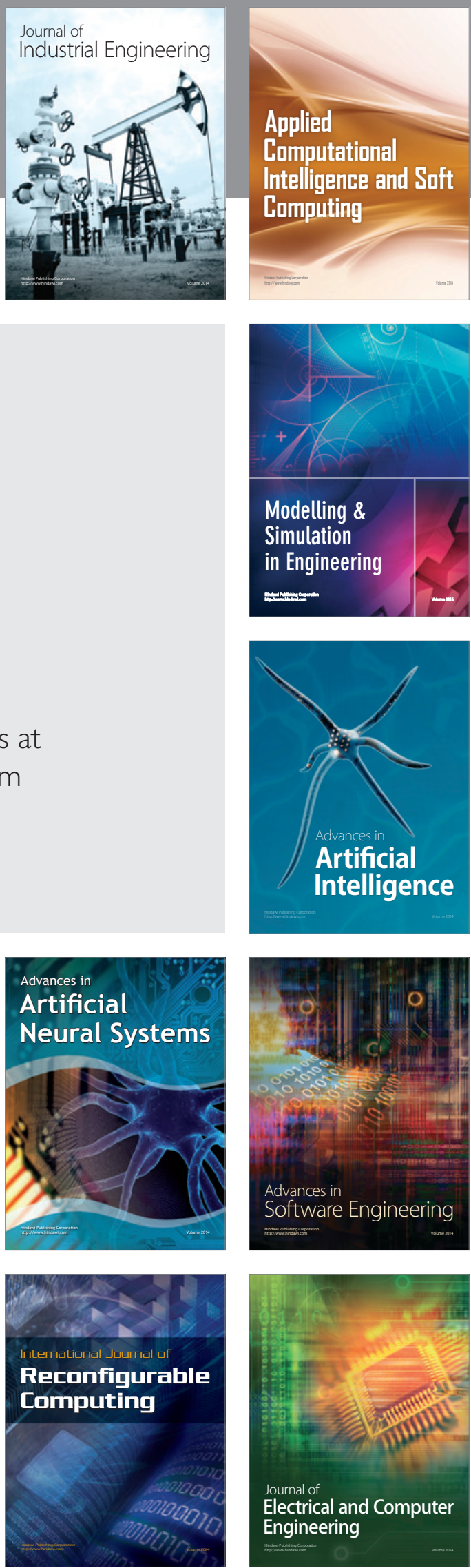